Paediatr. Paedolog. 2019 · 54 (Suppl 1):S16-S19 https://doi.org/10.1007/s00608-019-0659-9 Online publiziert: 12. März 2019

(c) Der/die Autor(en) 2019

\section{Claudia Wild}

Ludwig Boltzmann Institute for Health Technology Assessment (LBI-HTA), Wien, Österreich

\title{
Zugang zu teuren Medikamenten: Verantwortung der Politik
}

\author{
Wenn Kosten-Nutzen-Bewertungen als \\ Steuerungsinstrument nicht mehr greifen
}

Dieser Beitrag basiert auf einem Vortrag, der auf der 12. Jahrestagung der politischen Kindermedizin gehalten wurde. Er befasst sich damit, den kollektiven Widerstand auf europäischer Ebene gegen inakzeptable Preise - insbesondere, aber nicht nur für „orphan drugs“ - zu beschreiben. Es werden politische Strategien thematisiert, mit denen man sich gegen diese Preise wehren kann und muss. Insbesondere sollte der Mythos entzaubert werden, dass diese Medikamente derart teuer sein müssen. Die Europäische Union ist 1993 als Wirtschaftsraum gegründet worden und verficht seitdem sehr industriefreundliche Strategien. Vor diesem Hintergrund ist es umso mehr als deutliches Zeichen $\mathrm{zu}$ verstehen, wenn die Europäische Kommission selbst das Thema „Zugang zu teuren Therapien“ in die Hand nimmt und wahrnimmt, dass unsere Solidarsysteme in Gefahr sind, wenn derartige Preise bezahlt werden sollen.

Ich bin Mitglied in einem Expert Panel on Effective Ways of Investing in Health, das Politikberatungsdokumente für die Europäische Kommission (GD Sante) erarbeitet [1]. Die Fragestellung der europäischen Kommission an das Expertengremium war: Welche Möglichkeiten gibt es, tatsächlich innovative Medikamente, die auch einen Unterschied machen, zu fördern? Es handelt sich hier um eine kritische Fragestellung, da wir aus der Nutzenbewertung wissen, dass sehr wenige wirkliche Innovationen auf den $\mathrm{Me}$ dikamentenmarkt kommen. Eine weitere Frage der Europäischen Kommission war: Was kann getan werden, damit der Zugang zu innovativen Medikamenten nicht durch die sehr hohen Preise verwehrt wird und was ist - grosso modo - zu tun, damit wir unser solidarisches Gesundheitswesen für die nächste Generation erhalten?

Der Hintergrund der Problemstellung ist, dass sehr viele Medikamente nur marginale oder gar keine Effekte zeigen und trotzdem zugelassen werden. Wir beobachten ein hohes Ausmaß an Orphanisierung seit der begünstigten Zulassungsregelung. Das gilt nicht so sehr in der Kindermedizin, aber sehr stark für die Onkologie. Der Grund liegt darin, dass die Regularien zur Zulassung von „orphan drugs“ derart gute Bedingungen schaffen, dass inzwischen beinahe jede (onkologische) Erkrankung eine „orphan disease“ ist. Jedes dritte Medikament, das in der EU zugelassen wird, ist ein onkologisches Medikament (und davon sind wiederum die Hälfte „orphan Drugs"), während in anderen Bereichen keine Forschung passiert und auch keine Medikamente auf dem Markt erscheinen. Verschärft wird die Problematik durch die sehr unfaire Preisgestaltung. Aufgrund der exorbitant hohen Medikamentenpreise haben bereits die Niederlande während ihrer EU-Ratspräsidentschaft 2016 faire Medikamentenpreise als Themenschwerpunkt gesetzt. Das Thema wurde von der bulgarischen EU-Ratspräsidentschaft 2018 fortgeführt und diese Staffel hat dann Österreich im 2. Halbjahr 2018 während seines darauffolgenden Ratsvorsitzes übernommen.
Unser Schwesterinstitut in Deutschland, das Institut für Wirtschaftlichkeit und Qualität im Gesundheitswesen (IQWIG), führt im Rahmen des deutschen Gesetzes zur Neuordnung des Arzneimittelmarkts (AMNOG) eine Bewertung von beinahe allen Medikamenten durch, die auf dem deutschen und damit auch auf dem österreichischen Markt zugelassen werden. Gegenüber den bereits verfügbaren Medikamenten haben $56 \%$ der seit 2011 (bis 2017, $n=234$ ) zugelassenen Medikamente keinen $\mathrm{Zu}$ satznutzen. Nur $8 \%$ respektive $18 \%$ der Medikamente haben einen erheblichen respektive beträchtlichen Zusatznutzen und sind als echte Innovationen zu bezeichnen. Nusinersen $\left(\right.$ Spinraza $^{\circledR}$ ) fällt in diese $8 \%$ der Medikamente mit dem höchsten Zusatznutzen.

Nun haben wir uns zum Thema „Zugang zu Medikamenten“ in der bereits erwähnten Kommission den Kopf zerbrochen: Was kann getan werden, was passiert bereits und was kann über die nächsten 10 Jahre getan werden? Die Ideen setzen sich aus Vorschlägen aus allen Teilen der Welt zusammen, da wir in Österreich oder in der EU nicht allein mit diesem Thema sind. Auch Kanada, die USA und alle Entwicklungsländer sind genauso davon betroffen, ob sie Medikamente gegen Hepatitis $\mathrm{C}$ oder Aids-Medikamente, zu einem vernünftigen Preis erhalten. Letztendlich sind 8 Ideen aufgebracht worden, die ich vorstellen werde. 


\section{Größere Preis- und Kostentransparenz}

Die erste Lösungsmöglichkeit ist, dass genauer angeschaut wird, wie es eigentlich zur Preisbildung kommt. Wie kann es sein, dass ein Medikament 500.000 oder $300.000 €$ kostet? Ist das gerechtfertigt? Wenn man bei der Industrie nachfragt, hört man mehr oder weniger monoton die Wiederholung des Mythos, dass ausschließlich die Forschungskosten daran schuld seien - ohne aber detaillierte Belege vorlegen zu können (oder zu wollen). Wenn mein Gegenüber, dem ich eine Frage stelle, diese nicht beantwortet, muss ich versuchen, die Antworten selbst zu finden. Dementsprechend wurde begonnen, die einzelnen Phasen der Arzneimittelproduktion oder -erforschung aufzufächern und sich anzusehen, wo welche Kosten anfallen.

Die Vereinigten Staaten sind hier deutlich transparenter als Europa, da in den USA jede einzelne Bilanz eines börsennotierten Betriebs online gestellt werden muss. Es ist zwar ein hoch mühsames Unterfangen dem nachzugehen, aber letztendlich wissen wir, dass ungefähr $50 \%$ der Forschungskosten (konservativ geschätzt) im öffentlichen Bereich liegen, da die ganze Grundlagenforschung in Universitäten durchgeführt wird. Sobald ein Molekül identifiziert und patentiert ist, geht es in die erste Entwicklungsphase („proof of concept“). Diese wird meist von Biotech-Start-ups durchgeführt, die dieses Molekül weiterentwickeln und die erste Entwicklungsphase eines Arzneimittels einleiten. Hier gibt es eigene Patentscouts - das sind bereits Vertreter der Industrie, die beobachten, was auf den Universitäten geforscht wird und wo etwas in die Nähe der Anwendbarkeit rückt, um dann eben dieses Patent oder Lizenzen aufzukaufen. Ab diesem Zeitpunkt finden sich Informationen in börsennotierten Betrieben auch online in den Bilanzen. Davor kann in Forschungsberichten und -publikationen festgestellt werden, wer $\mathrm{zu}$ welchem Thema geforscht hat. Als Beispiel: Spinraza ${ }^{\circledR}$ wurde exklusiv mit Forschungsgeldern des NIH (US-National Institute of Health) erforscht, und zwar von der University of Massachusetts.
Dann wurde es von Ionis Pharmaceuticals aufgekauft und zuletzt von Biogen übernommen. An den Zulassungsstudien für die Food and Drug Administration (FDA) nahmen etwa 460 Kinder mit spinaler Muskelatrophie teil. Der öffentliche Sektor zahlt also zweimal: einmal für die Grundlagenforschung und einmal für den Medikamenteneinkauf. Der Gewinn erfolgreicher Forschung ist privatisiert.

Ziel der Nachforschung über Medikamentenentwicklung ist es einerseits, dem Mythos nachzugehen, ob wirklich die Forschungs- und Entwicklungskosten derart hoch sind, wie sie von der Pharmaindustrie dargestellt werden. Andererseits ist ein Ziel, sich genauer zu überlegen, ob es nicht andere Modelle der Arzneimittelentwicklung gäbe auch öffentliche Modelle, um jene Medikamente verfügbar zu haben, die wir dringend brauchen, wo tatsächlich „unmet need“ besteht. Diese Modelle gibt es, so hat beispielsweise die Initiative für Drugs for Neglected Diseases (DND) inzwischen 5 Medikamente zur Zulassung gebracht. Es handelt sich hierbei um Medikamente für vernachlässigte Krankheiten - also um genau die Erkrankungen in Entwicklungsländern, um die sich keiner kümmert, weil der Markt zwar da ist, aber keiner die Medikamente bezahlen kann. Darüber hinaus gibt es andere Initiativen, sogenannte Priority Setting Partnerships. Dabei geht es um „open innovation “ und „open science“, d. h. dass alles, was in öffentlichen Institutionen erforscht wird, auch öffentlich verfügbar ist, sodass darauf aufbauend eine öffentliche Arzneimittelentwicklung stattfinden kann. Aus diesen Beispielen ist es nachvollziehbar, was Arzneimittelentwicklung kostet und auch kosten kann.

Die zweite Idee bei der genauen Analyse der Phasen von Arzneimittelentwicklung ist, dass einzelne Phasen öffentlich übernommen werden, d. h. es wird eine Ausschreibung organisiert, so wie es im Augenblick in der Antibiotikaforschung passiert. Dort nimmt die Europäische Kommission 500 Mio. $€$ in die Hand und schreibt diese unter der Bedingung aus, dass innerhalb von 10 Jahren neue Antibiotika auf dem Markt erscheinen. Dasselbe könnte in anderen Produktgruppen gemacht werden, indem die Entwicklung bis zur Zulassung ausgeschrieben und bezahlt wird und ab der Zulassung das entwickelte Medikament nicht patentgeschützt und damit kostengünstig verfügbar ist. Auf dieser Ebene entstehen aufgrund des enormen Drucks - inzwischen sehr viele Ideen, wie Arzneimittelentwicklung anders stattfinden kann, ohne am Ende hohen Preisen ausgeliefert zu sein. Dieser Gewinn kommt ja letztendlich den Aktionären zugute und nicht dem öffentlichen Gesundheitswesen.

\section{Innovationen fördern, beispielsweise durch veränderten Patentschutz}

Die zweite Idee ist, dass der Patentschutz genauer betrachtet wird, weil der Patentschutz zwar schützt, aber nicht unbedingt Innovationen fördert. Wie bereits erwähnt, verdienen es $56 \%$ der neu zugelassenen Medikamente nicht, Innovation genannt zu werden, sind aber trotzdem patentgeschützt. Bei „orphan drugs“ kann der Patentschutz bis zu 20 Jahre betragen.

Der Anlassfall, der alle Länder aufgerüttelt hat, war letztendlich Sofosbuvir (Sovaldi ${ }^{\circledR}$ ). Die Lizenz für Sovaldi ${ }^{\circledR}$ wurde für $11 \mathrm{Mrd}$. $€$ von einem kleinen Unternehmen gekauft und diese $11 \mathrm{Mrd}$ € wurden innerhalb von 6 Monaten aufgrund der hohen Therapiekosten bereits wieder hereingespielt. Nun ist eine der (berechtigten) Argumentationen der Industrie, dass es Overheads bzw. Risikokapital geben muss, weil nicht jedes Medikament erfolgreich wird. Darüber, wie hoch die sogenannten Kapitalisierungskosten sein müssen, zerbrechen sich insbesondere Experten in Kanada den Kopf: d.h. darüber, ob 2-facher oder 5-facher Gewinn ausreicht, um das Risiko abzudecken. Das Ergebnis dieser Diskussionen ist, dass bei Blockbustern der Patentschutz eben nach 3 Jahren bereits ausläuft. Das heißt, hier geht es in Richtung Flexibilisierung des Patentschutzes.

Es gibt einzelne Präparate, die immer wieder ein Schlupfloch finden, um über viele Jahre hinweg Patente zu erneuern mit dem Ergebnis, dass der offizielle Patentschutz von 10 bis 12 Jahren, respektive bei „orphan drugs" von 20 Jahren, 
durchaus bis zu 40 Jahre lang ausgebeutet werden kann. Aus diesem Grund hat die Europäische Kommission im Jahr 2018 zwei voneinander unabhängige Gutachten in Auftrag gegeben, die untersuchen sollen, wie der Patentschutz anders geregelt werden könnte.

\section{Entwicklung von Methoden, um den sozialen Wert von Medikamenten zu messen}

Der dritte Bereich umfasst eher Lösungsmöglichkeiten auf niedrigschwelliger Ebene. Die Nutzenbewertungen im Rahmen des Health Technology Assessment (HTA) sind methodisch an ihre Grenze gestoßen. So viel Nutzen gegenüber derart hohen Preisen kann kein Medikament erzeugen. Bei $400.000 €$ für Spinraza $^{\circledR}$ ist eine Kosten-Nutzen-Bewertung nicht mehr möglich. Deswegen wird überlegt und daran gearbeitet, dass statt Kosteneffektivitätsgrenzen stärker andere Methoden zur Sozialverträglichkeit und Bezahlbarkeit von Preisen erprobt werden. Das ist jedoch noch in einem sehr frühen methodischen Entwicklungsstadium.

\section{Erhöhung der Marktmacht in Preisverhandlungen}

Ein Lösungsansatz, bei dem die ersten Umsetzungsschritte schon gegangen worden sind, ist, dass die Marktmacht erhöht wird. Wir sind in Österreich ja besonders kleinteilig, indem wir 9 Bundesländer haben. Aber auch Österreich selbst ist ein kleiner Markt. Deswegen haben sich innerhalb von Europa Länder zusammengefunden, die ungefähr gleich finanzkräftig sind. Dies sind auf der einen Seite die südlichen Länder (Valetta) und auf der anderen Seite die Nordländer (FiNoSe). Wir sind in einem Verbund mit den Beneluxländern (BeNeLuxA), in dem wir gemeinsam über die Preise der besonders teuren $\mathrm{Me}$ dikamente verhandeln. Im Augenblick gibt es noch rechtliche Barrieren, sodass Österreich bis jetzt nur Observer bei den Verhandlungen zum Einkauf von teuren Arzneimitteln von Belgien und den Niederlanden sein konnte. Belgien hat etwa 11 Mio. Einwohner und die Niederlande

Paediatr. Paedolog. 2019 · 54 (Suppl 1):S16-S19 https://doi.org/10.1007/s00608-019-0659-9

(c) Der/die Autor(en) 2019

\section{Wild}

\section{Zugang zu teuren Medikamenten: Verantwortung der Politik. Wenn Kosten-Nutzen-Bewertungen als Steuerungsinstrument nicht mehr greifen}

\section{Zusammenfassung}

Der Anstieg der Arzneimittelausgaben aufgrund neuer, hochpreisiger innovativer Arzneimittel stellt die Gesundheitssysteme unter den derzeitigen institutionellen Rahmenbedingungen vor finanzielle Herausforderungen. Die Erkenntnis, dass der derzeitige Wachstumspfad nicht unbegrenzt fortbestehen kann, erfordert die Notwendigkeit neuer Strategien, um den Zugang für Patienten zu Innovationen zu sichern und um die Gesundheitssysteme nachhaltig finanzieren zu können. In einem Bericht des EU-Expertengremiums „Effective Ways of Investing in Health" kommen einige Vorschläge für Politikansätze zum Vorschlag: Höhere Preis- und Kostentransparenz; Revision des

\begin{abstract}
Patentrechts und der Marktexklusivität; Entwicklung von Methoden zur Messung des sozialen Werts von Arzneimitteln, bessere Belohnung für bessere therapeutische Wirksamkeit; Zahlungssysteme, die (erfolgreiche) Behandlungen erstatten, statt Pillen einzukaufen; Erprobung nichtlinearer Refundierungen; Ausübung von Marktmacht bei Preisverhandlungen; Schaffung von Dialogplattformen, an denen alle relevanten Stakeholder beteiligt sind.
\end{abstract}

Schlüsselwörter Arzneimittel · Seltene Erkrankungen · Gesundheitspolitik · Kosten · Health Technology Assessment $\cdot$ Pharmaindustrie

\section{Access to Expensive Drugs: The Responsibility of Policy. When Cost-Benefit Analyses are no Longer Suitable as a Management Tool}

\section{Abstract}

The growth of pharmaceutical expenditure due to new high-cost innovative medicines, under the current institutional framework, creates financial challenges for health systems. The recognition that the current path of growth cannot continue indefinitely leads to the need for new policies that secure patient access to innovation and for health systems to remain financially sustainable. In a report by the EU "Expert Panel on Effective Ways of Investing in Health" some suggestions for political approaches are proposed: greater price and cost transparency; revision of the patent law and market exclusivity; development of methodologies to measure the social value of pharmaceutical products; better rewards for better therapeutic effectiveness; payment systems that reimburse (successful) treatments, instead of buying pills; trial of non-linear reimbursements; exercising of market power in price negotiations; creation of dialogue platforms with the participation of all relevant stakeholders.

\section{Keywords}

Drugs · Rare illnesses · Health policy · Costs . Health technology assessment (HTA) Pharmaceutical industry etwa 17 Mio. Einwohner. Diese beiden Länder haben bereits zusammen Preise für Spinraza ${ }^{\circledR}$ verhandelt.

\section{Höheren therapeutischen Nutzen fördern}

Die nächste Lösungsidee ist, darüber nachzudenken was - abseits von $\mathrm{Pa}$ tentschutz - getan werden kann, damit innovativere Medikamente erforscht werden oder mehr innovative Medika- mente zugelassen werden. Eine zentrale Idee ist es, die Latte für Zulassungen durch die European Medicines Agency (EMA) höher zu legen. So gäbe es beispielsweise die Möglichkeit, dass durch die Zulassungsbehörde festgelegt wird, dass 3 Wochen Lebensverlängerung in der Onkologie zu wenig sind, also die Lebensverlängerung größer sein muss, bevor ein Medikament zugelassen wird. Diese Idee ist noch in einem frühen Diskussionsstadium und es wird natürlich 
dauern, die Zulassungsauflagen einer Behörde (die sich allerdings zu 85\% durch die Pharmaindustrie finanziert) zu verändern.

\section{Weiterentwicklung von Bezahlungsmodellen, die Wirksamkeit belohnen}

Die nächste Idee ist, nicht einfach eine Pille einzukaufen und pro Stück zu bezahlen, sondern, dass dann bezahlt wird, wenn sich eine Wirksamkeit einstellt. Das bedeutet letztlich, dass sehr teure Medikamente nur unter Dokumentation verabreicht werden dürfen: Spinraza ${ }^{\circledR}$ wäre ein Kandidat dafür. So wäre ein entsprechendes Bezahlungsmodell, dass nur für jene Kinder Kosten anfallen, die auf Spinraza $^{\circledR}$ ansprechen, und für alle anderen Kinder, die ohne Wirkung therapiert werden, keine Kosten anfallen. Diese Bezahlungsmodelle gibt es bereits in vielen Ländern, nicht aber in Österreich. Hier gibt es in erster Linie Rabattierungsmodelle.

In Kanada und den skandinavischen Ländern wird hingegen über sogenannte Starting und Stopping Rules nachgedacht. Bei Spinraza ${ }^{\circledR}$ beispielsweise wird überlegt, wie lange man abwartet, bis eine Patientin oder ein Patient auf die medikamentöse Therapie anspricht und wann die Therapie abgebrochen wird, wenn die Patientin oder der Patient nicht darauf anspricht. Letztlich geht es um flexiblere Finanzierungsmodelle. Das ist natürlich aufwendig, weil viel dokumentiert werden muss. Bei Spinraza ${ }^{\circledR}$ muss mit Videoaufnahmen dokumentiert werden, wie sich das Kind motorisch entwickelt. Videoaufnahmen müssen zwischen Kindern verglichen werden. Wir wissen, wie unterschiedlich sich gesunde Kinder entwickeln. Daher ist es keine trivial zu lösende Frage, ob ein Medikament wirkt oder nicht.

Dabei besteht natürlich das Risiko, dass dort, wo ein Medikament wirkt, statt $500.000 €$ auf einmal 1,5 Mio. $€$ bezahlt werden, um die Kosten (den erwarteten Gewinn) abzudecken, wo das Medikament nicht gewirkt hat. Es gilt niemals $\mathrm{zu}$ vergessen, dass wir es mit gewinnorientierten Unternehmen $\mathrm{zu}$ tun haben. Dies zeigt, dass jedes neue Bezahlungs- modell in seinen Konsequenzen vor der Einführung gründlich durchdacht werden sollte.

\section{Nichtlineare Bezahlungsschemata}

Dann gibt es auch diese Idee von nichtlinearen Bezahlungsschemata, d. h. die Preise für ein Medikament unterscheiden sich beispielsweise zwischen Indikationen.

\section{Dialogplattformen aller relevanten Stakeholder aufbauen}

Der letzte Lösungsansatz ist, dass die unterschiedlichen Player im System stärker miteinander reden, was aber bereits stattfindet. Es geht dabei um den sogenannten Scientific Advice oder Early Dialogue. Hierbei sprechen Pharmafirmen bereits frühzeitig mit der Zulassungsbehörde und mit HTA darüber, wie Studien aufgesetzt werden sollen und was harte Endpunkte sind, die auch für HTA akzeptabel sind. Hier sehen wir, dass man sich zwischen Zulassungsbehörde und HTA bezüglich Nutzenbewertung schnell einig ist, wenn es um die genaue Population geht, dass man sich aber tendenziell weniger einig ist, wenn es darum geht, was der Komparator (die Vergleichsintervention) sein könnte und welches die relevanten Endpunkte sind.

Ein weiterer Vorschlag wäre, dass stärker bei den Postmarket-SurveillanceDatenerhebungen kooperiert wird. Viele Medikamente werden ja „conditionally approved“, da zum Zeitpunkt der Zulassung noch zu wenig über die Wirkung bekannt ist. Hier verlangt die EMA nach der Zulassung Datenerhebungen. Und diese Datenerhebungen sollten mit dem übereinstimmen, was in HTA verlangt wird, sodass nur für das bezahlt wird, was auch wirklich wirksam ist.

\section{Conclusio}

Die Take-home-Message ist, dass man mit den herkömmlichen Methoden von HTA nicht mehr weiterkommt und es daher politische Lösungen geben muss.

Diese Lösungen sind aufgrund des Kostendrucks rasch anzugehen.

\section{Korrespondenzadresse

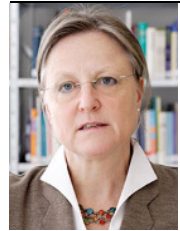 \\ (c) LBI-HTA \\ Priv. Doz. Dr. phil Claudia Wild \\ Ludwig Boltzmann Institute for Health Technology Assessment (LBI-HTA) Garnisongasse 7/20, 1090 Wien, Österreich claudia.wild@hta.lbg.ac.at}

Priv. Doz. Dr. phil Claudia Wild Claudia Wild ist seit der Gründung des Ludwig Boltzmann Instituts für Health Technology Assessment (LBI-HTA) im Jahr 2006 Institutsleiterin. Von 1989 bis 2006 war sie wissenschaftliche Mitarbeiterin am Institut für Technikfolgen-Abschätzung an der Österreichischen Akademie der Wissenschaften (ÖAW) und mit dem Aufbau und der Leitung des Arbeitsbereichs Health Technology Assessment (HTA) betraut. Davor war sie am Lehrstuhl für Kommunikations- und Politikwissenschaften an der Universität Nürnberg tätig. Ihr Studium der Kommunikationswissenschaft und Psychologie an der Universität Wien und der Politikwissenschaft an der Ohio University/USA schloss sie 1985 mit einer Promotion ab. Im Jahr 2009 habilitierte sie in Sozialmedizin (Medizinische Universität Graz) zum Thema "Ressourcenallokation im Gesundheitswesen". Im Jahr 2020 wird das LBI-HTA in das Austrian Institute for HTA (AIHTA) überführt

\section{Einhaltung ethischer Richtlinien}

Interessenkonflikt. C. Wild gibt an, dass kein Interessenkonflikt besteht.

Dieser Beitrag beinhaltet keine von den Autoren durchgeführten Studien an Menschen oder Tieren.

Open Access Dieser Artikel wird unter der Creative Commons Namensnennung 4.0 International Lizenz (http://creativecommons.org/licenses/by/4.0/deed. de) veröffentlicht, welche die Nutzung, Vervielfältigung, Bearbeitung, Verbreitung und Wiedergabe in jeglichem Medium und Format erlaubt, sofern Sie den/die ursprünglichen Autor(en) und die Quelle ordnungsgemäß nennen, einen Linkzur Creative Commons Lizenz beifügen und angeben, ob Änderungen vorgenommen wurden.

Hinweis des Verlags. Der Verlag bleibt in Hinblick auf geografische Zuordnungen und Gebietsbezeichnungen in veröffentlichten Karten und Institutsadressen neutral.

\section{Literatur}

1. Expert Panel on „Effective ways of investing in health" (2018) Innovative payment models for high-cost innovative medicine. https://ec.europa. eu/health/expert_panel/sites/expertpanel/files/ docsdir/opinion_innovative_medicines_en.pdf 